\title{
APLICAÇÃO DE DOSES DE ZINCO, VIA SOLO, NA BANANEIRA "PRATA ANÃ" (AAB) IRRIGADA, NO NORTE DE MINAS GERAIS ${ }^{(1)}$
}

\author{
Enilson de Barros Silva ${ }^{(2)}$, Múcio Mágno de Melo Farnezi ${ }^{(3)}$, Paulo \\ Jorge de Pinho ${ }^{(4)}$, Maria Geralda Vilela Rodrigues ${ }^{(5)}$ \& Janice \\ Guedes de Carvalho ${ }^{(6)}$
}

\begin{abstract}
RESUMO
Foi realizado um experimento de campo no município de Nova Porteirinha, no Norte de Minas Gerais, em Latossolo Vermelho-Amarelo distrófico, com os objetivos de avaliar a resposta da bananeira 'Prata Anã' à aplicação de doses de Zn via solo e estimar os níveis críticos de Zn no solo e nas folhas. Foram aplicadas quatro doses

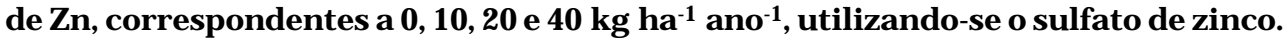
Os tratamentos foram dispostos no delineamento em blocos casualizados com três repetições, totalizando 36 parcelas experimentais. Foram avaliados a produtividade de frutos, o teor de Zn no solo (Mehlich-1 e DTPA a pH 7,3) e o teor foliar durante dois anos de cultivo. A produtividade de frutos aumentou com as doses de $\mathrm{Zn}$ aplicadas no solo, atingindo, na média de dois anos de cultivo, $22,2 \mathrm{t} \mathrm{ha}^{-1}$ com aplicação da dose de $4,1 \mathrm{~kg} \mathrm{ha}^{-1}$ ano $^{-1}$ de $\mathrm{Zn}$. O nível crítico médio de Zn no solo pelos extratores Mehlich-1 e DTPA foi de 14,5 e 5,2 $\mathrm{mg} \mathrm{dm}^{-3}$, respectivamente, e, para $\mathrm{Zn}$ foliar, de $\mathbf{1 5 , 8} \mathrm{mg} \mathrm{kg}^{-1}$, nas condições edafoclimáticas da região Norte de Minas Gerais.
\end{abstract}

Termos de indexação: Musa sp., nutrição da bananeira, Latossolo, análise foliar, nível crítico.

\footnotetext{
(1) Trabalho desenvolvido com suporte financeiro do Banco do Nordeste. Recebido para publicação em novembro de 2004 e aprovado em julho de 2007.

(2) Professor Adjunto do Departamento de Agronomia da Universidade Federal dos Vales do Jequitinhonha e Mucuri - UFVJM. Rua da Glória 187, CEP 39100-000 Diamantina (MG). E-mail: ebsilva@ufvjm.edu.br.

(3) Mestrando em Produção Vegetal, UFVJM. E-mail: muciomagno@yahoo.com.br

${ }^{(4)}$ Doutorando do Departamento de Ciências do Solo, Universidade Federal de Lavras - UFLA. Caixa Postal 37, CEP 37200-000 Lavras (MG). E-mail: pinhopj@yahoo.com.br

${ }^{(5)}$ Pesquisadora da EPAMIG/CTNM. Caixa Postal 112, CEP 39527-000 Nova Porteirinha (MG). E-mail: magevr@epamig.br

(6) Professora Titular do Departamento de Ciências do Solo, UFLA. E-mail: janicegc@ufla.br
} 


\title{
SUMMARY: APPLICATION OF ZINC DOSES VIA SOIL TO IRRIGATED BANANA "PRATA ANÃ" (AAB) IN NORTHERN MINAS GERAIS, BRAZIL
}

\begin{abstract}
A field experiment was conducted in Nova Porteirinha, $M G$, Brazil, in a dystrophic Red Yellow Latossol, to detect the response of the banana tree 'Prata Anã' to Zn doses through soil and critical $\mathrm{Zn}$ levels in soil and leaves. Four $\mathrm{Zn}$ doses were applied, corresponding to 0, 10,20 and $40 \mathrm{~kg} \mathrm{ha}^{-1}$ yearl, using zinc sulfate as source. The treatments were arranged in a randomized block design, with three repetitions per block, totaling 36 experimental plots. The following traits were evaluated: fruit yield, soil $\mathrm{Zn}$ content (Mehlich-1 and DTPA pH 7.3) and leaf Zn, in two cropping years. The fruit yield increased

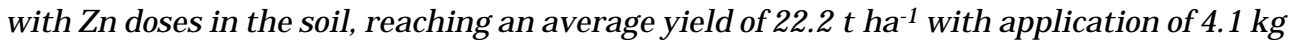
$\mathrm{Zn} \mathrm{ha}^{-1}$ year ${ }^{-1}$ in two crops. The mean critical level of soil $\mathrm{Zn}$ by the extractors Mehlich-1 and $D T P A$, was 14.5 and $5.2 \mathrm{mg} \mathrm{dm}^{-3}$, respectively and $15.8 \mathrm{mg} \mathrm{kg}^{-1}$ for leaf $\mathrm{Zn}$, in the edaphoclimatic conditions of northern Minas Gerais.
\end{abstract}

Index terms: Musa sp., banana nutrition, Oxisol, leaf analysis, critical level.

\section{INTRODUÇÃO}

A região Norte de Minas Gerais vem despontando como um grande pólo frutícola do Brasil. A cultura da banana é a sua principal atividade agrícola, ocupando 10.000 ha (Dantas Filho, 2000); 90 \% dessa área é ocupada pela cultura da banana "Prata Anã". Nessa região, a bananeira encontra condições edafoclimáticas favoráveis para obter elevada produtividade, com frutos de boa qualidade, além de exercer importante papel socioeconômico.

A produção de banana é afetada por fatores internos da planta, como os genéticos, e também por fatores externos, como condições de clima, solo e manejo. É uma cultura muito exigente em nutrientes, devido à sua elevada produtividade, podendo alcançar de 50 a 70 t ha $^{-1}$ ano $^{-1}$ (Silva et al., 1999). Segundo Lopez \& Espinosa (1995), a nutrição é fator de produção de extrema importância para a bananeira, devido à alta eficiência dessas plantas em produzir grande quantidade de biomassa em curto período de tempo.

Bananeiras que apresentam deficiência de Zn produzem frutos tortos, pequenos e com coloração verde-pálida e folhas jovens em forma lanceolada, com coloração avermelhada (Lahav \& Turner, 1983; Moreira, 1999).

A disponibilidade do Zn no solo para as plantas tem sido estimada por diversos extratores. Abreu \& Raij (1996), Menezes (1998) e Nascimento et al. (2002) comentaram que o extrator DTPA foi o que apresentou maior sensibilidade à mudança de $\mathrm{pH}$ do solo. Nascimento et al. (2002) verificaram que a solução extratora Mehlich-1, que é um extrator ácido, é menos indicada para avaliar a disponibilidade de $\mathrm{Zn}$, pois extrai formas pouco disponíveis às plantas ligadas a sítios de adsorção específica, como $\mathrm{Zn}$ ligado a hidróxidos e carbonatos.
No Brasil, as melhores correlações entre os teores de $\mathrm{Zn}$ em solos e o seu teor nas plantas têm sido obtidas pelo método em que se emprega a solução DTPA como extrator (Cantarella et al., 1998); a solução Mehlich-1 apresenta resultado igual ou inferior ao DTPA de correlação com os teores de Zn nas plantas (Abreu, 1995). As classes de fertilidade para Zn extraído por DTPA sugeridas por Raij et al. (1996), para tipos de solos e culturas, em geral, são: baixa fertilidade (menos do que $0,6 \mathrm{mg} \mathrm{dm}^{-3}$ de $\mathrm{Zn}$ ), média (de 0,6 a $1,2 \mathrm{mg} \mathrm{dm}^{-3} \mathrm{de} \mathrm{Zn}$ ), alta (mais do que $1,2 \mathrm{mg} \mathrm{dm}^{-3} \mathrm{de}$ Zn). Para Alvarez V. et al. (1999), para o Estado de Minas Gerais, são muito baixos os teores menores do que $0,4 \mathrm{mg} \mathrm{dm}^{-3}$; baixos, de 0,5 a 0,9 ; médios, de $1,0 \mathrm{a}$ 1,5 ; suficientes, de 1,6 a 2,2; e altos, maiores do que 2,2 $\mathrm{mg} \mathrm{dm}^{-3} \mathrm{de} \mathrm{Zn}$ extraído pelo extrator Mehlich-1.

Moreira (1999) recomenda, para bananeira, a aplicação de 9,$0 ; 7,5 ; 4,5 ; 3,0 ;$ e $1,5 \mathrm{~kg} \mathrm{ha}^{-1} \mathrm{ano}^{-1} \mathrm{de}$ $\mathrm{Zn}$ quando os teores pela análise química do solo (extração com DTPA a pH 7,3) forem < 0,5; entre 0,6 e 1,1 ; entre 1,2 e 1,3 ; entre 1,4 e 1,5 ; e $>1,5 \mathrm{mg} \mathrm{dm}^{-3}$ de $\mathrm{Zn}$, respectivamente.

Não existe valor do teor de $\mathrm{Zn}$ foliar que seja considerado adequado e possa ser usado como padrão para todos os locais de cultivo e cultivares; entretanto, considera-se que uma planta está deficiente quando esses teores encontram-se abaixo de $17 \mathrm{mg} \mathrm{kg}^{-1} \mathrm{e}$ que está bem nutrida quando os teores foliares encontramse entre 17 e $50 \mathrm{mg} \mathrm{kg}^{-1}$ de Zn (Malavolta, 1980). Silva $\&$ Rodrigues (2001) observaram que o teor médio de Zn foliar de um levantamento feito, sem avaliação da produtividade, em 1.099 amostras de folhas de bananais do Norte de Minas foi de 19,2 $\mathrm{mg} \mathrm{kg}^{-1}$.

Silva et al. (2002) consideram, para bananeiras cultivadas no Norte do Estado de Minas Gerais, o valor de referência de $14 \mathrm{mg} \mathrm{kg}^{-1}$ nas folhas como aquele abaixo do qual as plantas se encontram deficientes. Os autores, com base na faixa de suficiência obtida 
em bananais da mesma região, verificaram que o teor de Zn apresentou-se dentro da faixa de 14 a $25 \mathrm{mg} \mathrm{kg}^{-1}$ nas folhas e inferior à sugerida por Prezotti (1992) e Malavolta et al. (1997), de 20 a $50 \mathrm{mg} \mathrm{kg}^{-1}$. Silva \& Rodrigues (2001), utilizando a faixa de suficiência sugerida pelos últimos autores, avaliaram que $72 \%$ dos bananais estavam deficientes em Zn. Essa discrepância entre as faixas de suficiência estabelecidas para os bananais do Norte de Minas e as faixas apresentadas na literatura ocasionou os equívocos que ocorreram na interpretação dos teores de nutrientes foliares, levando a recomendações inadequadas de adubação para os bananais da região (Silva et al., 2002).

O objetivo deste trabalho foi determinar a produtividade média de frutos, a dose de $\mathrm{Zn}$ e o nível crítico deste elemento no solo para os extratores Mehlich-1 e DTPA pH 7,3 e nas folhas da bananeira "Prata Anã" (AAB) irrigada e cultivada, em um Latossolo Vermelho-Amarelo distrófico da região Norte do Estado de Minas Gerais.

\section{MATERIAL E MÉTODOS}

O experimento foi realizado na área pertencente à EPAMIG/CTNM, localizada na Colonização II do Perímetro Irrigado do Gorutuba, Nova PorteirinhaMG, ao norte do Estado de Minas Gerais. A altitude da sede do município é de $500 \mathrm{~m}$, com latitude de $15^{\circ} 47^{\prime} \mathrm{S}$, longitude de $43^{\circ} 17^{\prime} \mathrm{E}$ e precipitação pluvial média anual de $800 \mathrm{~mm}$, sendo o clima classificado com Aw, segundo Köppen (Antunes, 1986).

O solo da área experimental foi classificado como Latossolo Vermelho-Amarelo distrófico (Embrapa, 2006), de textura média. Para caracterização do solo foram coletadas amostras compostas na profundidade de 0 a $20 \mathrm{~cm}$, que, depois de secas ao ar e passadas em peneira de $2 \mathrm{~mm}$, foram analisadas química $\mathrm{e}$ fisicamente (Quadro 1).

O plantio das mudas foi realizado em outubro de 2001, e estas completaram três ciclos sucessivos de produção, sendo o primeiro do plantio até a colheita, que foi de um ano (outubro de 2001 a setembro de 2002); o segundo e terceiro ciclos foram de um ano (outubro de 2002 a setembro de 2003), que correspondeu a dois anos de cultivo do experimento. A cultivar foi plantada "Prata Anã", obtida por cultura de tecidos, no espaçamento de $3,0 \times 2,7 \mathrm{~m}$ (1.235 plantas ha $\left.{ }^{-1}\right)$.

A calagem da área não foi necessária, uma vez que a saturação por bases estava acima da recomendada para bananeira (Alvarez V. \& Ribeiro, 1999), que é de $70 \%$ (Quadro 1). A adubação fosfatada de plantio foi de $142 \mathrm{~kg} \mathrm{ha}^{-1} \mathrm{de} \mathrm{P}_{2} \mathrm{O}_{5}$ na forma de superfosfato simples, e a cada ano de produção, na forma de MAP (fosfato monoamônico). Após o pegamento das mudas, a adubação potássica foi de
Quadro 1. Características químicas e físicas do solo na profundidade de 0 a $20 \mathrm{~cm}$ antes da implantação do experimento

\begin{tabular}{|c|c|}
\hline Característica & Valor \\
\hline $\mathrm{pH}$ em $\mathrm{H}_{2} \mathrm{O}(1: 2,5)^{(1)}$ & 6,9 \\
\hline$P\left(m g \mathrm{dm}^{-3}\right)^{(1)}$ & 17 \\
\hline $\mathrm{K}\left(\mathrm{mg} \mathrm{dm}^{-3}\right)^{(1)}$ & 250 \\
\hline $\mathrm{Ca}^{2+}\left(\mathrm{cmol}_{\mathrm{c}} \mathrm{dm}^{-3}\right)^{(1)}$ & 5,0 \\
\hline $\mathrm{Mg}^{2+}\left(\mathrm{cmol}_{\mathrm{c}} \mathrm{dm}^{-3}\right)^{(1)}$ & 1,1 \\
\hline $\mathrm{Al}^{3+}\left(\mathrm{cmol}_{\mathrm{c}} \mathrm{dm}^{-3}\right)^{(1)}$ & 0,1 \\
\hline $\mathrm{V}(\%)^{(2)}$ & 84 \\
\hline M.O. $\left(\mathrm{g} \mathrm{kg}^{-1}\right)^{(3)}$ & 24 \\
\hline Zn - Mehlich-1 $\left(\mathrm{mg} \mathrm{dm}^{-3}\right)^{(1)}$ & 8,0 \\
\hline $\mathrm{Zn}-$ DTPA pH 7,3 $\left(\mathrm{mg} \mathrm{dm}^{-3}\right)^{(1)}$ & 2,7 \\
\hline Areia $\left(\mathrm{g} \mathrm{kg}^{-1}\right)^{(4)}$ & 530 \\
\hline Silte $\left(\mathrm{g} \mathrm{kg}^{-1}\right)^{(4)}$ & 270 \\
\hline Argila $\left(\mathrm{g} \mathrm{kg}^{-1}\right)^{(4)}$ & 200 \\
\hline
\end{tabular}

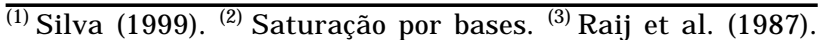
${ }^{(4)}$ Embrapa (1997).

$720 \mathrm{~kg} \mathrm{ha}^{-1} \mathrm{ano}^{-1}$ de $\mathrm{K}_{2} \mathrm{O}$ na forma de cloreto de potássio. A adubação nitrogenada foi de $326 \mathrm{~kg} \mathrm{ha}^{-1}$ $\mathrm{ano}^{-1}$ de $\mathrm{N}$ na forma de uréia. A adubação com $\mathrm{B}$ foi feita com aplicação de $2,0 \mathrm{~kg} \mathrm{ha}^{-1} \mathrm{ano}^{-1}$ na forma de bórax, dividido em três aplicações, via solo.

O preparo do solo, o plantio, os tratos culturais e o controle de pragas e doenças seguiram as recomendações de Souto et al. (1997). A irrigação foi feita por microaspersão, com aplicação diária de uma lâmina equivalente a $80 \%$ da evaporação do tanque classe A, de acordo com Costa et al. (1999).

No experimento foram aplicadas no solo, por cova, quatro doses de $\mathrm{Zn}$, equivalentes a $0,10,20$ e $40 \mathrm{~kg} \mathrm{ha}^{-1}$ $a^{-1}$. O delineamento experimental foi em blocos casualizados, com três repetições, totalizando 36 parcelas experimentais. A parcela útil constou de quatro plantas centrais em $32,4 \mathrm{~m}^{2}$, e a parcela total, de 18 plantas em $145,8 \mathrm{~m}^{2}$. A área total do experimento foi de $5.248,8 \mathrm{~m}^{2}$.

A produtividade de frutos de banana foi avaliada pela pesagem das pencas dos cachos por parcela útil, colhidos quatro meses após início da emissão da inflorescência, em cada ciclo sucessivo de produção. O Zn foi extraído do solo com as soluções de Mehlich1 e DTPA a pH 7,3, sendo dosado por espectrometria de absorção atômica (Silva, 1999) em amostras compostas coletadas na profundidade de 0 a $20 \mathrm{~cm}$, na emissão da inflorescência de cada planta da parcela útil de cada ciclo de produção. Os teores foliares de $\mathrm{Zn}$ foram determinados na terceira folha a partir do 
ápice, retirada no início da emissão da inflorescência (Malavolta et al., 1997) de cada ciclo de produção. A mineralização foi realizada por digestão nitroperclórica e a dosagem de Zn, por espectrometria de absorção atômica segundo método descrito por Malavolta et al. (1997).

Os dados de produtividade de frutos e teor de $\mathrm{Zn}$ no solo e nas folhas foram submetidos à análise de variância a $5 \%$ de significância. Para produtividade de frutos, foram ajustadas regressões polinomiais, para dois anos de cultivo, em função das doses de $\mathrm{Zn}$ aplicadas no solo. A partir das equações obtidas para produtividade de frutos, estimaram-se as doses de $\mathrm{Zn}$ necessárias para obtenção de $95 \%$ da produtividade máxima, sendo considerada como de máxima eficiência econômica.

Com base nas doses de Zn associadas à máxima eficiência econômica e nas regressões que relacionam os teores de $\mathrm{Zn}$ no solo recuperado pelos extratores Mehlich-1 e DTPA a pH 7,3 e nas folhas da bananeira em função das doses de $\mathrm{Zn}$ aplicadas no solo, estimaram-se os níveis críticos de Zn para bananeira "Prata Anã" plantada em Latossolo Vermelho-Amarelo distrófico nas condições climáticas do Norte de Minas Gerais, em dois anos de cultivo.

\section{RESULTADOS E DISCUSSÃO}

A produtividade de frutos de banana aumentou segundo um modelo raiz quadrático, com o incremento das doses de $\mathrm{Zn}$ aplicadas via solo nos dois anos de cultivo (Figura 1). A produtividade para $95 \%$ da máxima estimada com a equação ajustada entre a produtividade de frutos e as doses de $\mathrm{Zn}$ aplicadas ao solo (Figura 1) foi obtida com a dose de 3,3 e 4,9 $\mathrm{kg} \mathrm{ha}^{-1}$ ano ${ }^{-1}$, respectivamente, para o primeiro e segundo anos de cultivo, com média de $4,1 \mathrm{~kg} \mathrm{ha}^{-1} \mathrm{ano}^{-1}$ de $\mathrm{Zn}$ aplicado no solo e produtividade média de frutos de banana, nos dois anos, igual a 22,2 $\mathrm{t} \mathrm{ha}^{-1}$.

A diminuição da produtividade de frutos de banana em função de doses excessivas de $\mathrm{Zn}$ é provavelmente decorrente da menor translocação de fotoassimilados, pois há interferência do excesso de Zn no carregamento no floema, impedindo a translocação de fotoassimilados para as principais partes da planta (Fávaro, 1992). Para Malavolta et al. (1997), a toxidez se manifesta pela diminuição da área foliar, seguida de clorose, e pode aparecer na planta inteira um pigmento pardoavermelhado, provavelmente devido a um fenol. Outra conseqüência da toxidez, ou excesso de $\mathrm{Zn}$, é a diminuição da absorção de P. No xilema de algumas espécies de plantas que apresentam sintomas de toxidez acumulam-se tampões com $\mathrm{Zn}$, que dificultam a ascensão da seiva bruta.

A disponibilidade de $\mathrm{Zn}$ para as plantas é diretamente relacionada às frações trocáveis e

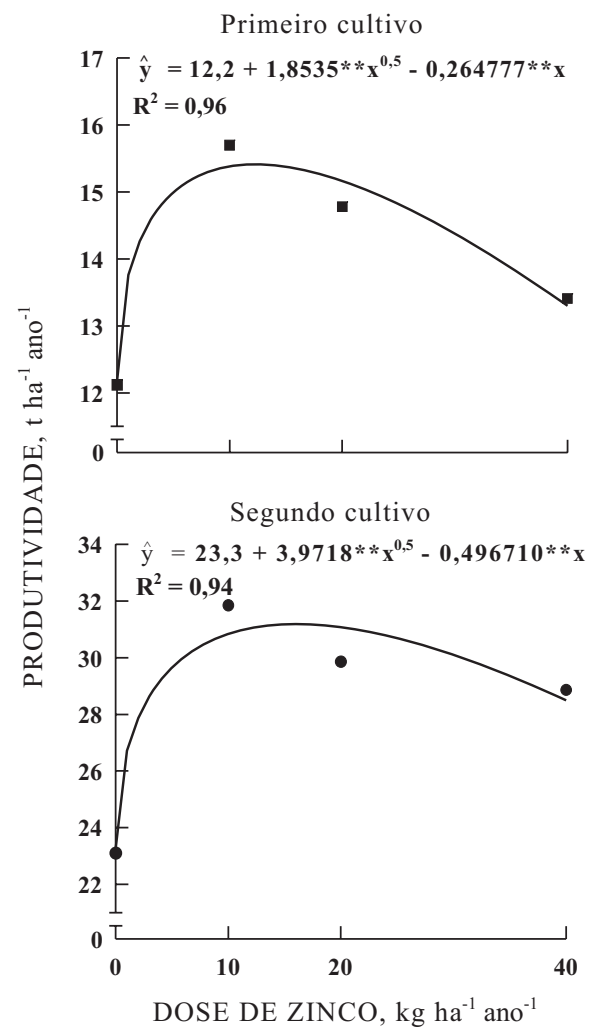

Figura 1. Produtividade de frutos de banana "Prata Anã" irrigada em função de doses de zinco, via solo, em dois cultivos. ( ${ }^{* *}$ significativo a $1 \%$ pelo teste de t).

inversamente ao $\mathrm{pH}$ do solo (Nascimento et al., 2002). A forma trocável de $\mathrm{Zn}$ no solo é predominante em solução em pH abaixo de 6,0; mas, acima desse valor, a concentração de $\mathrm{Zn}^{2+}$ decresce acentuadamente (Sanders, 1983). Pela análise inicial do solo, o pH é igual a 6,9 (Quadro 1), o que proporciona drástica diminuição dos teores de Zn trocável, devido à sua precipitação em forma de compostos de baixa solubilidade, como carbonatos e hidróxidos, conforme Barrow (1985), diminuindo a quantidade de $\mathrm{Zn}$ disponível no solo para absorção pela bananeira.

No diagnóstico da deficiência de Zn, é importante realizar a análise do solo antecipadamente para fazer possíveis correções na adubação com Zn para bananeira "Prata Anã", a fim de minimizar prejuízos futuros na produtividade. O nível crítico de $\mathrm{Zn}$ no solo (camada de 0 a $20 \mathrm{~cm}$ ) para $95 \%$ da produtividade máxima obtido pelo extrator Mehlich-1 (Figura 2) foi de 12,4 e $8,8 \mathrm{mg} \mathrm{dm}^{-3}$ e, para o DTPA (Figura 3), de 4,1 e $3,3 \mathrm{mg} \mathrm{dm}^{-3}$, para o primeiro e segundo anos de cultivo, respectivamente. Os níveis críticos de Zn no solo são elevados tanto pelo extrator Mehlich-1, com base em valores definidos por Alvarez V. et al. (1999), quanto pelo DTPA, com base em valores definidos por Raij et al. (1996) e Moreira (1999). Os níveis críticos de $\mathrm{Zn}$ disponível foram maiores com o extrator 


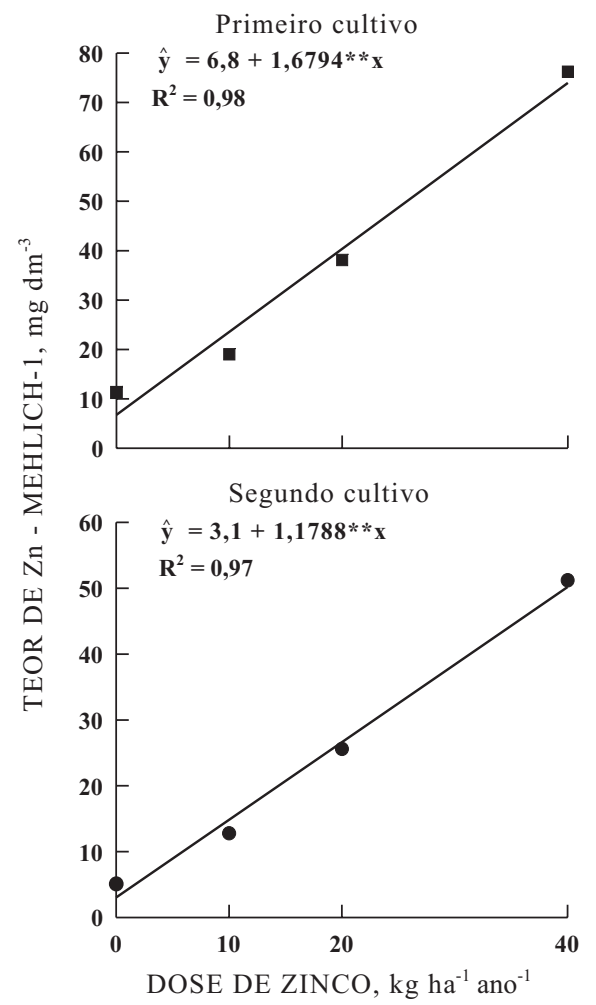

Figura 2. Teor de Zn no solo extraído por Mehlich-1 em função de doses de zinco, via solo, em dois cultivos. ( ${ }^{* *}$ significativo a $1 \%$ pelo teste de $\left.t\right)$.

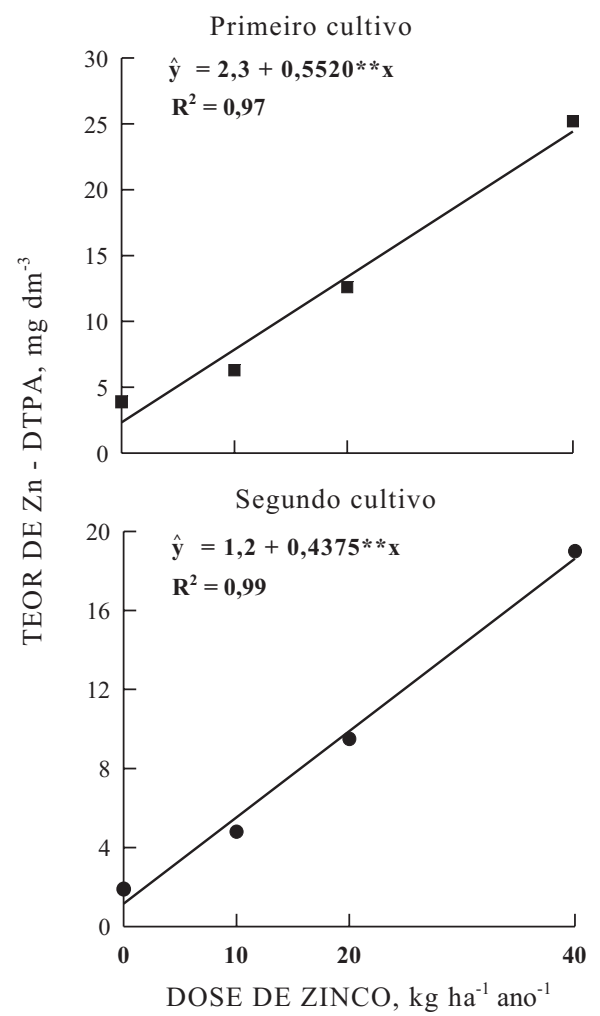

Figura 3. Teor de Zn no solo extraído por DTPA em função de doses de zinco, via solo, em dois cultivos. (**significativo a $1 \%$ pelo teste de $t)$.
Mehlich-1 (ácido) em relação ao DTPA (agente quelante), com a mesma tendência observada por Nascimento et al. (2002), o que se deve ao mecanismo de atuação dos extratores, com maior recuperação de Zn pelos extratores que atacam os colóides por dissolução ácida.

Em relação ao teor foliar de Zn (Figura 4), utilizouse o mesmo procedimento adotado para o $\mathrm{Zn}$ disponível no solo. O nível crítico de $\mathrm{Zn}$ foliar foi de $14,1 \mathrm{e}$ $13,5 \mathrm{mg} \mathrm{kg}^{-1}$ para o primeiro e segundo cultivos, respectivamente, estando abaixo dos preconizados por Prezotti (1992) e Malavolta et al. (1997) e dentro da faixa de suficiência definida por Silva et al. (2002) em avaliação nutricional dos bananais do Norte de Minas Gerais. A discrepância entre o nível crítico foliar de Zn encontrado na região Norte de MG e a faixa de suficiência de Zn proposta por Prezotti (1992) e Malavolta et al. (1997) para bananeira se deve à utilização, por esses autores, de métodos de diagnose nutricionais estabelecidos em condições edafoclimáticas diferentes das que são observadas na região Norte do Estado de Minas Gerais.

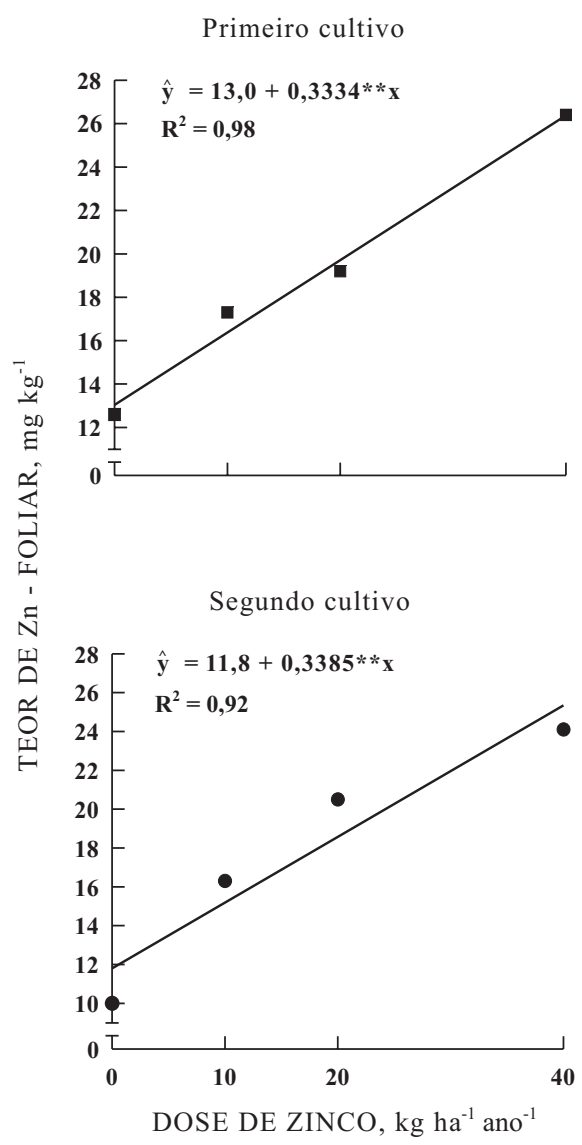

Figura 4. Teor de $\mathrm{Zn}$ foliar em função de doses de zinco, via solo, em dois cultivos. (**significativo a $1 \%$ pelo teste de $t$ ). 


\section{CONCLUSÃO}

A produtividade de frutos de banana "Prata Anã" irrigada, em Latossolo Vermelho-Amarelo distrófico da região Norte de Minas Gerais, aumentou com o incremento das doses de $\mathrm{Zn}$ aplicadas no solo, atingindo produtividade média de $22,2 \mathrm{t} \mathrm{ha}^{-1}$ com a dose de $4,1 \mathrm{~kg} \mathrm{ha}^{-1}$ ano $^{-1}$, com níveis críticos de Zn disponível no solo iguais a $10,6 \mathrm{mg} \mathrm{dm}^{-3}$ (Mehlich-1) e $3,7 \mathrm{mg} \mathrm{dm}^{-3}$ (DTPA) e, na planta (foliar), de $13,8 \mathrm{mg} \mathrm{kg}^{-1}$.

\section{AGRADECIMENTOS}

Ao Banco do Nordeste e ao Centro Tecnológico do Norte de Minas, da Empresa de Pesquisa Agropecuária do Estado de Minas Gerais (EPAMIG/CTNM), pelo apoio e pela cooperação.

\section{LITERATURA CITADA}

ABREU, C.A. Análise de solo para micronutrientes: Tema de reuniões de laboratórios. B. Inf. SBCS, 20:128-130, 1995.

ABREU, C.A. \& RAIJ, B. van. Efeito da reação do solo no zinco extraído pelas soluções de DTPA e Mehlich-1. Bragantia, 55:357-363, 1996.

ALVAREZ V., V.H. \& RIBEIRO, A.C. Calagem. In: RIBEIRO, A.C.; GUIMARÃES, P.T.G. \& ALVAREZ V., V.H., eds. Recomendações para o uso de corretivos e fertilizantes em Minas Gerais. Viçosa, MG, Comissão de Fertilidade do Solo do Estado de Minas Gerais, 1999. p.43-60.

ALVAREZ V., V.H.; NOVAIS, R.F.; BARROS, N.F.; CANTARUTTI, R.B. \& LOPES, A.S. Interpretação dos resultados das análises de solos. In: RIBEIRO, A.C.; GUIMARÃES, P.T.G. \& ALVAREZ V., V.H., eds. Recomendações para o uso de corretivos e fertilizantes em Minas Gerais. Viçosa, MG, Comissão de Fertilidade do Solo do Estado de Minas Gerais, 1999. p.25-32.

ANTUNES, F.Z. Caracterização climática do Estado de Minas Gerais. Inf. Agropec., 12:9-13, 1986.

BARROW, N.J. Reaction of anions and cations with variablecharge soils. Adv. Agron., 38:183-230, 1985.

CANTARELLA, H.; RAIJ, B. van \& QUAGGIO, J.A. Soil and plant analyses for lime and fertilizer recommendations in Brazil. Comm. Soil Sci. Plant Anal., 29: 1691-1706, 1998.

COSTA, E.L.; MAENO, P. \& ALBUQUERQUE, P.E.P. Irrigação da bananeira. Inf. Agropec., 20:67-72, 1999.

DANTAS FILHO, L.E. Agricultura mineira 1998. Belo Horizonte, GCEA, 2000. 347p.

EMPRESA BRASILEIRA DE PESQUISA AGROPECUÁRIA EMBRAPA. Centro Nacional de Pesquisa de Solos. Manual de métodos de análise do solo. Brasília, SPI, 1997. 212p.

EMPRESA BRASILEIRA DE PESQUISA AGROPECUÁRIA EMBRAPA. Centro Nacional de Pesquisa de Solos. Sistema brasileiro de classificação de solos. Brasília, Produção de Informação, 2006. 306p.
FÁVARO, J.R.A. Crescimento e produção de Coffea arabica L. em resposta à nutrição foliar de zinco, na presença de cloreto de potássio. Viçosa, MG, Universidade Federal de Viçosa, 1992. 91p. (Tese de Mestrado)

LAHAV, E. \& TURNER, D.W. Banana nutrition. Bern, International Potash Institute, 1983. 62p. (IPI Bulletin, 7)

LOPEZ, M.A. \& ESPINOSA, M.J. Manual de nutrition y fertilización del banano. Quito, Instituo de la Potasa y el Fósforo, 1995. 82p.

MALAVOLTA, E. Elementos de nutrição mineral de plantas. Piracicaba, Ceres, 1980. 254p.

MALAVOLTA, E.; VITTI, G.C. \& OLIVEIRA, S.A. Avaliação do estado nutricional das plantas: Princípios e aplicações. 2.ed. Piracicaba, POTAFOS, 1997. 319p.

MENEZES, A.A. Disponibilidade de zinco, para milho, pelos extratores Mehlich-1, Mehlich-3 e DTPA. Viçosa, MG, Universidade Federal de Viçosa, 1998. 147p. (Tese de Mestrado)

MOREIRA, R.S. Banana: Teoria e prática de cultivo. Campinas, Fundação Cargill, 1999. CD-ROM.

NASCIMENTO, C.W.A.; FONTES, R.L.F.; NEVES, J.C.L. \& MELÍCIO, A.C.F.D. Fracionamento, dessorção e extração química de zinco em Latossolos. R. Bras. Ci. Solo, 26:599606, 2002.

PREZOTTI, C. Recomendações de calagem e adubação para o Estado do Espírito Santo. $3^{\mathrm{a}}$ aproximação. Vitória, EMCAPAR, 1992. 73 p. (Circular Técnica, 12)

RAIJ, B.van; CANTARELLA, H.; QUAGGIO, J.A. \& FURLANI, A.N.C., eds. Recomendações de adubação e calagem para o Estado de São Paulo. Campinas, Instituto Agronômico/ Fundação IAC, 1996. 285 p. (IAC Boletim Técnico, 100).

RAIJ, B.van; QUAGGIO, J.A.; CANTARELLA, H.; FERREIRA, M.E.; LOPES, A.S. \& BATAGLIA, O.C. Análise química do solo para fins de fertilidade. Campinas, Fundação Cargill, 1987. 170p.

SANDERS, J.R. The effects of $\mathrm{pH}$ on the total and frees ionic concentrations of manganese, zinc and cobalt in soil solutions. J. Soil Sci., 34:315-323, 1983.

SILVA, E.B. \& RODRIGUES, M.G.V. Levantamento nutricional dos bananais da Região Norte de Minas Gerais pela análise foliar. R. Bras. Frutic., 23:695-698, 2001.

SILVA, F.C. Manual de análises químicas de solos, plantas e fertilizantes. Brasília, Embrapa, 1999. 370p.

SILVA, J.T.A.; BORGES, A.L. \& MALBURG, J.L. Solos, adubação e nutrição da bananeira. Inf. Agropec., 20:21$36,1999$.

SILVA, J.T.A.; BORGES, A.L.; DIAS, M.S.C.; COSTA, E.L. \& PRUDÊNCIO, J.M. Diagnóstico nutricional da bananeira "Prata Anã" para o norte de Minas. Belo Horizonte, EPAMIG, 2002. 16 p. (Boletim Técnico, 70)

SOUTO, R.F.; RODRIGUES, M.G.V.; ALVARNGA, C.D.; SILVA, J.T.A.; MAENO, P. \& GONZAGA, V. Sistema de produção para a cultura da banana prata anã no Norte de Minas. Belo Horizonte, EPAMIG, 1997. 32p. (Boletim Técnico, 48) 